\title{
Periodicities in data observed during the minimum and the rising phase of solar cycle 23; years 1996-1999
}

\author{
S. Zięba, J. Masłowski, A. Michalec, and A. Kułak
}

Astronomical Observatory, Jagiellonian University, ul Orla 171, 30-244 Kraków, Poland

Received 18 July 2000 / Accepted 11 July 2001

\begin{abstract}
Three types of observations: the daily values of the solar radio flux at 7 frequencies, the daily international sunspot number and the daily Stanford mean solar magnetic field were processed in order to find all the periodicities hidden in the data. Using a new approach to the radio data, two time series were obtained for each frequency examined, one more sensitive to spot magnetic fields, the other to large magnetic structures not connected with sunspots. Power spectrum analysis of the data was carried out separately for the minimum (540 days from 1 March 1996 to 22 August 1997) and for the rising phase (708 days from 23 August 1997 to 31 July 1999) of the solar cycle 23. The Scargle periodograms obtained, normalized for the effect of autocorrelation, show the majority of known periods and reveal a clear difference between the periodicities found in the minimum and the rising phase. We determined the rotation rate of the "active longitudes" in the rising phase as equal to $444.4 \pm 4 \mathrm{nHz}(26.0 \pm 0.3)$. The results indicate that appropriate and careful analysis of daily radio data at several frequencies allows the investigation of solar periodicities generated in different layers of the solar atmosphere by various phenomena related to the periodic emergence of diverse magnetic structures.
\end{abstract}

Key words. Sun: activity - Sun: radio radiation - Sun: magnetic fields - Sun: rotation

\section{Introduction}

The study of periodicities in different solar data is important for the understanding of solar magnetic activity. For over a decade many authors have reported various periods other than those at $11 \mathrm{yr}$ and 27 days, of which the one near 154 days is the best known (Rieger et al. 1984; Dennis 1985; Bogart \& Bai 1985; Lean \& Brueckner 1989; Bai \& Cliver 1990; Dröge et al. 1990; Pap et al. 1990; Carbonell \& Ballester 1990, 1992; Bai \& Sturrock 1991, 1993; Kile \& Cliver 1991; Bouver 1992; Oliver et al. 1998; Ballester et al. 1999). This investigation was designed to show that accurate daily radio observations can be very useful for studying the solar periodicities generated in different layers of the solar atmosphere, and in different phases of the solar cycle. We used a new approach to the daily measured radio fluxes which allow us to separate to some extent the radio emission generated in the strong magnetic fields of active regions from that emitted by large but weaker magnetic structures. All the solar data from the minimum and the rising phase of solar cycle 23 were processed separately using the Scargle periodogram technique (Scargle 1982). The statistical significance levels of all the periods found were estimated through the FAP (false alarm probability - the probability that the given periodogram value $z$

Send offprint requests to: A. Michalec,

e-mail: michalec@oa.uj .edu.pl is generated by noise), as well as by a Monte Carlo approach to the periodograms obtained (Horne \& Baliunas 1986; Bai 1992b; Özgüç \& Ataç 1994; Oliver \& Ballester 1995).

Papers published by Das \& Chatterjee (1996); Das \& Nag $(1998,1999)$ presented some periods in radio data without any discussion about their significance level. Our analysis of radio observations however, gives the majority of the known periods, reveals a clear difference between periodicities observed in two phases of the solar cycle and shows that daily measured radio fluxes at various frequencies are very useful for the systematic study of solar periodicities observed in the different layers of the solar atmosphere.

\section{Observations}

The radio fluxes at the four lowest frequencies 405, $810,1215,1620 \mathrm{MHz}$ are from our new accurate observations, which we began on 1 March 1996 (http://www.oa.uj.edu.pl.). Every day we measure the total solar radio flux at ten frequencies within the decimeter range of wavelengths using the 8-m radiotelescope built in 1995 at the Cracow Astronomical Observatory (Zięba et al. 1996). The international sunspot numbers $(I S N)$, the Stanford mean solar magnetic field $(M M F)$ and radio fluxes at the frequencies 2800, 4995 and $8800 \mathrm{MHz}$ were 
Table 1. Parameters of the two different models of the solar radio emission used to eliminate the basic component $B$ from the daily observed flux $F_{i}$.

\begin{tabular}{|c|c|c|c|c|c|c|c|}
\hline \multicolumn{8}{|c|}{ Linear model $F_{i}=B+h(I S N)_{i}$} \\
\hline frequency in $\mathrm{MHz}$ & 405 & 810 & 1215 & 1620 & 2800 & 4995 & 8800 \\
\hline$B$ - basic component [su] & $22.9 \pm .2$ & $39.8 \pm .3$ & $48.2 \pm .4$ & $53.5 \pm .4$ & $67.3 \pm .5$ & $117.0 \pm .5$ & $199.4 \pm .1$ \\
\hline$h$ - radio flux production & $0.138 \pm .003$ & $0.337 \pm .005$ & $0.508 \pm .007$ & $0.611 \pm .007$ & $0.785 \pm .008$ & $0.633 \pm .008$ & $0.752 \pm .017$ \\
\hline $\begin{array}{l}\% \text { of the data variance } \\
\text { explained by the model }\end{array}$ & 71.0 & 78.6 & 81.9 & 85.7 & 87.5 & 83.5 & 61.6 \\
\hline Boltzman formula model & \multicolumn{7}{|c|}{$\overline{F_{i}}=B_{i}+h_{\mathrm{B}}(I S N)_{i}=A_{2}+\left(A_{1}-A_{2}\right) /\left(1+\exp \left(\left(t_{i}-t_{\circ}\right) / \Delta t\right)\right)+h_{\mathrm{B}}(I S N)_{i}$} \\
\hline frequency in $\mathrm{MHz}$ & 405 & 810 & 1215 & 1620 & 2800 & 4995 & 8800 \\
\hline parameters of $A_{1}[\mathrm{su}]$ & $22.1 \pm .5$ & $38.9 \pm .8$ & $48.0 \pm 1$ & $53.1 \pm 1.2$ & $67.4 \pm 2$ & $117.8 \pm 1.4$ & $200.5 \pm 7.6$ \\
\hline the Boltzman $A_{2}[\mathrm{su}]$ & $31.4 \pm .5$ & $62.5 \pm .7$ & $75.0 \pm .9$ & $78.9 \pm 1.1$ & $100.7 \pm 1.8$ & $137.1 \pm 1.5$ & $292.5 \pm 7.1$ \\
\hline sigmoidal $\quad t_{\circ}[$ days $]$ & $807 \pm 15$ & $855 \pm 7$ & $879 \pm 8$ & $880 \pm 10$ & $926 \pm 12$ & $962 \pm 11$ & $1064 \pm 20$ \\
\hline$\Delta t[$ days $]$ & $132 \pm 13$ & $111 \pm 7$ & $80 \pm 7$ & $91 \pm 9$ & $106 \pm 12$ & $45 \pm 12$ & $143 \pm 16$ \\
\hline$h_{\mathrm{B}}-$ radio flux production & $0.067 \pm .004$ & $0.154 \pm .006$ & $0.291 \pm .008$ & $0.408 \pm .010$ & $0.542 \pm .012$ & $0.487 \pm .012$ & $0.261 \pm .025$ \\
\hline $\begin{array}{l}\% \text { of the data variance } \\
\text { explained by the model }\end{array}$ & 81.0 & 91.8 & 91.4 & 91.5 & 92.5 & 87.5 & 79.3 \\
\hline
\end{tabular}

taken from Solar Geophysical Data bulletins (1996-1999). We added to our radio observations those at higher frequencies for two reasons. Firstly, we wanted to examine periodicities of the radio emission in a wide enough radio band to analyse the radio flux coming from a large part of the solar atmosphere and secondly to compare our observations with those of others.

It is trivial to form the time series from the daily values of the sunspot numbers and the mean magnetic field, but if we want to use the radio data for the study of magnetic activity, we must first eliminate from the observed daily flux (free from bursts) the thermal emission which comprises the majority of the daily measured value. This can be done through a procedure in which the flux observed on day $t_{i}, F_{i}$ is divided between the thermal, almost constant component (often called the basic component $-B)$ and the slowly varying component $(S V C)$, whose value changes every day and is generated by mechanisms dependent on the magnetic field. It is usually assumed that this component is proportional to a certain daily index of activity, for example $(I S N)_{i}$ and then the daily flux $F_{i}$ can be described by the following linear formula: $F i=B+(S V C)_{i}=B+h(I S N)_{i}$, where $h$ is the production of the radio flux from the spot with $I S N=1$ (Krüger 1979).

The assumption that $B$ is constant over the large time intervals is rather strong and cannot be accepted, especially at a time when the level of radio flux rises systematically. Here we propose a new approach in which the basic component $B$ is not constant over the given time interval but changes every day according to the Boltzman sigmoidal formula:

$B_{i}=A_{2}+\left(A_{1}-A_{2}\right) /\left(1+\exp \left(\left(t_{i}-t_{\circ}\right) / \Delta t\right)\right)$

where $A_{1}, A_{2}, t_{\circ}, \Delta t$ are the parameters and $t_{i}$ is the day number.

Then the observed daily flux $F_{i}=B_{i}+h_{\mathrm{B}}(I S N)_{i}$, and $h_{\mathrm{B}}$ has a similar interpretation to $h$. To determine the above parameters we used the observed radio data and daily sunspot numbers over the whole time interval investigated, 1 March 1996-31 July 1999 (1248 days).

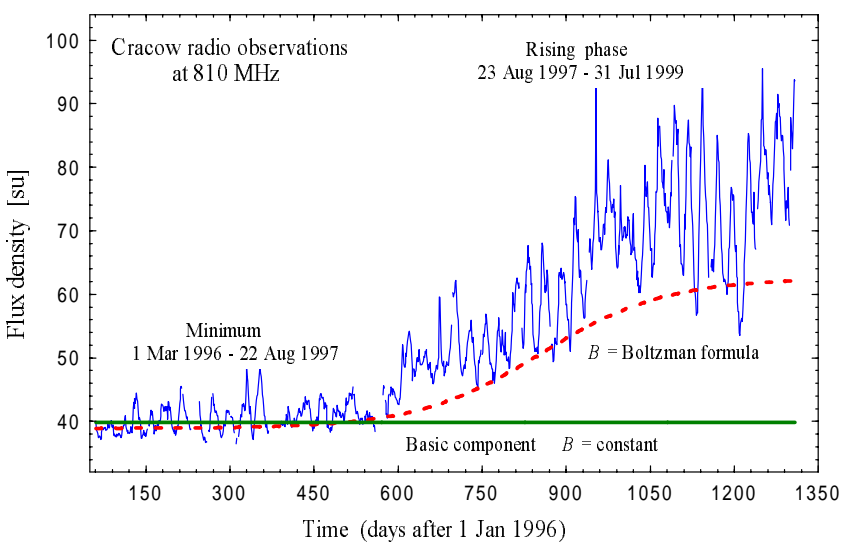

Fig. 1. Daily values of the radio flux at $810 \mathrm{MHz}$ observed from Cracow. The horizontal solid line shows the constant value of the basic component $B=39.8$ su resulting from the linear formula, while the dashed curve shows values of the basic component calculated from the best fitted parameters, $A_{1}=38.9 \mathrm{su}$, $A_{2}=62.5 \mathrm{su}, t_{\circ}=855$ days, $\Delta t=111$ days according to the Boltzman formula $B_{i}=A_{2}+\left(A_{1}-A_{2}\right) /\left(1+\exp \left(\left(t_{i}-t_{\circ}\right) /(\Delta t)\right)\right.$. The division into the minimum and rising phase is also indicated.

The best fit values of these parameters are shown in Table 1. The difference between the two models is clearly seen, especially for four frequencies 405, 810, 1215, $8800 \mathrm{MHz}$. To demonstrate this we present in Fig. 1, as an example, the daily values of the radio flux at $810 \mathrm{MHz}$ observed from Cracow as well as the calculated values of the basic component $B$ and $B_{i}$. The data in this figure also explain our division into the minimum and the rising phase.

Thus, in our approach to the radio data we create two time series from the observation at each frequency. The first, the $S V C$ (slowly varying component) time series, consists of diurnal values calculated as the difference between the daily observed flux and the daily value of the basic component computed from our model, $(S V C)_{i}=$ $F_{i}-B_{i}$. The second, the $R R E$ (radio residual emission) time series describes the every day difference between the radio observations and our model of the daily radio flux, 

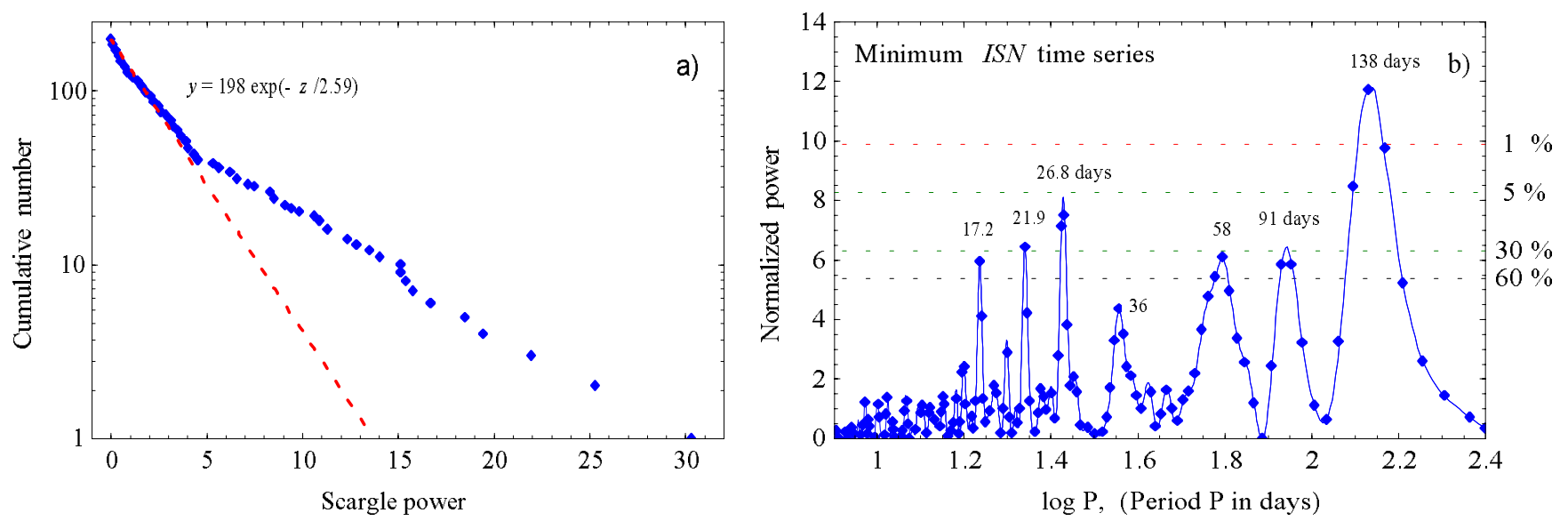

Fig. 2. a) The cumulative distribution function of the Scargle power for the original, minimum $I S N$ time series. The vertical axis is the number of frequencies whose power exceeds $z$. The straight line is the best fit to the points for values of power lower than 5. b) The normalized periodogram of the original, minimum $I S N$ time series with $F A P$ significance levels indicated.

$(R R E)_{i}=F_{i}-B_{i}-h_{\mathrm{B}}(I S N)_{i}$. Taking the time series $S V C$ and $R R E$, we can analyse cyclic variations of those magnetic structures which modified the observed radio emission. However, the $S V C$ series are more sensitive to spot magnetic fields, while the $R R E$ series are sensitive to large magnetic structures not connected with sunspots.

\section{Periodogram analysis}

The search for periodicities in all the time series was performed by calculating the Scargle normalized periodograms $P_{N}(\omega)$ (Scargle 1982). This technique (see Horne \& Baliunas 1986) has several advantages over the conventional fast Fourier transformation method and provides, through $F A P$, a simple estimate of the significance of the height of a peak in the power spectrum. However, the $F A P$ value is easy to calculate only for that time series for which the successive data are independent. In our case, the all analysed time series were prepared from the daily values of the different solar indices which are not independent but correlated with a characteristic correlation time of a week (Oliver \& Ballester 1995). Therefore, for all our FAP calculations used to estimate the statistical significance of a peak $z$, in the Scargle power spectrum, we applied the formula $F A P=1-[1-\exp (-z / k)]^{N}=$ $1-\left[1-\exp \left(-z_{m}\right)\right]^{N}$, where $N$ is the number of independent frequencies, $k$ is the normalization factor due to data correlation and $z_{m}=z / k$ the normalized power (Bai \& Cliver 1990; Bai 1992b). To determine the normalization factor $k$ for the given time series we followed the procedure described by Bai \& Cliver (1990). The key step of this procedure is the choice of a spectral window as well as the number of independent frequencies. Since we would like to analyse naturally limited time series connected with two different phases of the solar cycle over the largest possible range of periods, we took the interval 43 to $1447 \mathrm{nHz}$ (8270 days) as the spectral window for all the investigated time series. The shortest period, 8 days, is connected with a possible range of correlations of the analysed data (see Tables 2 and 3 where the autocorrelation coeficients with the lag $=1$ day and lag $=7$ days are given). The longest periods, 270 days, results from the actual length of the minimum time series equal to 540 days. The number of totally independent frequencies inside the chosen window is given by the value of the independent Fourier spacing, $\Delta f_{\text {ifs }}=1 / T$, where $T$ is the time span of the data (Scargle $1982)$. In the case of our time series, $T_{\mathrm{M}}=540$ days and $\Delta f_{\text {ifsM }}=21.433 \mathrm{nHz}$ for the minimum, while for the rising phase $T_{\mathrm{R}}=708$ days and $\Delta f_{\text {ifsR }}=16.348 \mathrm{nHz}$. However, de Jager (1987) has shown by Monte-Carlo simulations that the Fourier powers taken at intervals of one-third of the independent Fourier spacing are still statistically independent. Thus, we accepted the numbers 198 and 259 as the numbers of independent frequencies in the chosen window for the minimum and the rising phase time series, respectively. To illustrate the method used for determination of the normalization factor $k$, we will process one of our time series step by step; as an example we take the $I S N$ time series in the minimum phase. First, we calculate the Scargle power normalized by the variance of the data, (Horne \& Baliunas 1986) for all 198 independent frequencies which allows us to construct the graph presented in Fig. 2a. This shows the cumulative number of frequencies for which the Scargle power exceeds a certain value $z$. Then we fit all values of power $z<5$ to the equation $y=198 \exp (-z / k)$, which gives the value of the normalization factor $k$ equal 2.59 , since when $\exp (-z / k) \ll 1$ the formula for $F A P$ reduces to $F A P=N \exp (-z / k)$. Finally, we normalize the power spectrum once more by dividing the Scargle power by 2.59 to obtain the normalized periodogram for which $F A P$ values are easily calculated. Therefore, if we substitute $z=30.54, k=2.59$, and $N=198$ in the $F A P$ formula we get the normalized power $N P=z_{\mathrm{m}}=11.79$ and $F A P=0.0015$ for the highest peak in the $I S N$ minimum time series periodogram. Figure $2 \mathrm{~b}$ presents this normalized periodogram of the minimum $I S N$ series together with $F A P$ significance levels obtained from $F A P$ formula with $N=198$ and $k=2.59$. 

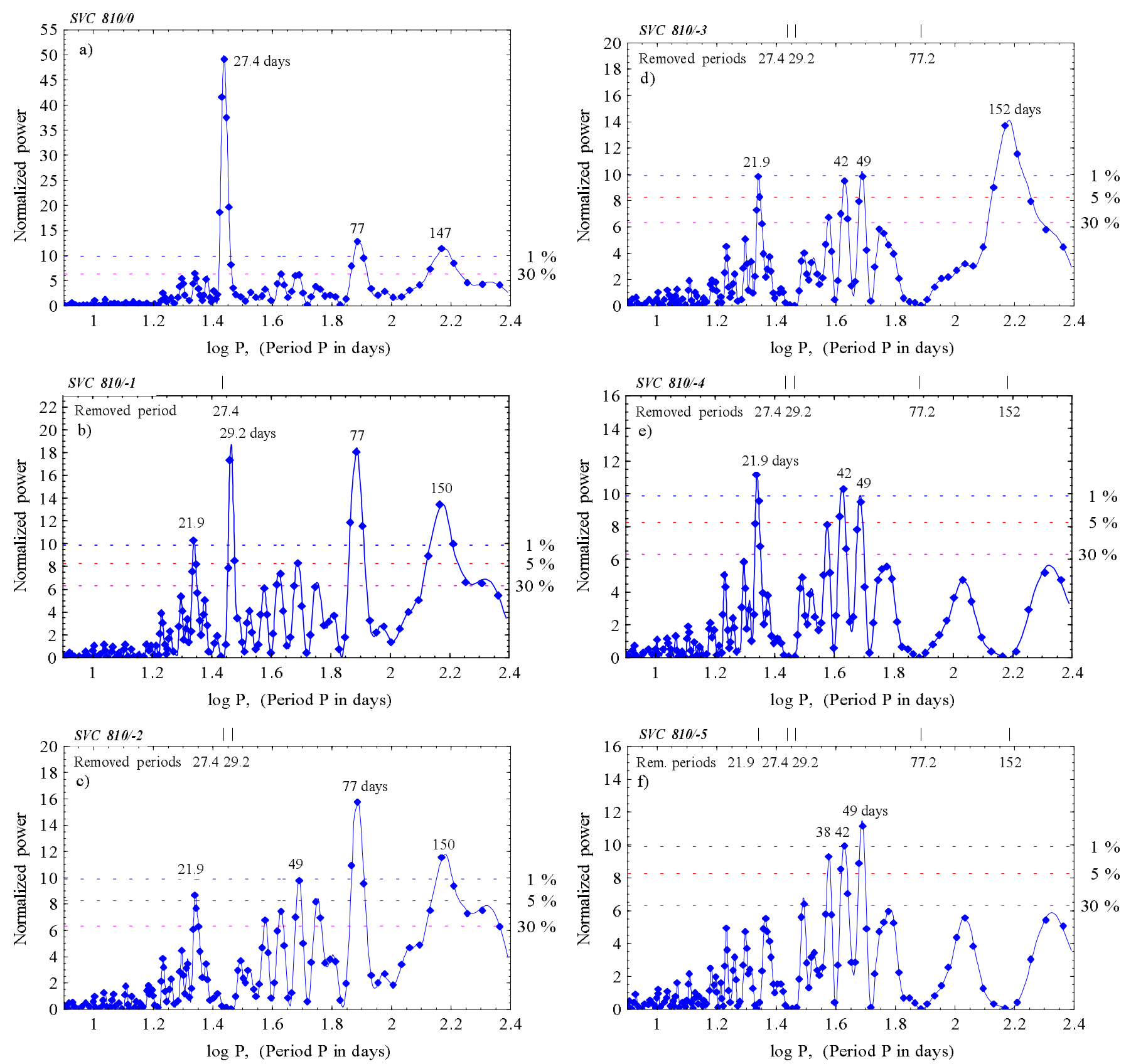

Fig. 3. a) The normalised periodogram of the minimum, original $S V C 810 / 0$ time series. b), c), ... same as a) but recalculated after successively removing from the original data one, two, and more sine curves having periods with peaks whose FAP values are smaller than $0.5 \%$. In each graph the removed periods are indicated at the top. The dashed lines show $F A P$ significance levels.

Since peaks in a periodogram may arise from aliasing or other phenomena not present in Gaussian noise (e.g., spectral leakage arising from the spacing of the data and from the finite length of the time series), the $F A P$ values alone, are insufficient for establishing whether or not strong peaks in a periodogram are indeed real periodicities in the time series. Also, some small peaks present in the original periodogram can be real in the case when the normalized factor $k$ would be too large in consequence of treating real periods as noise. We test for genuine peaks by recomputing the periodogram after randomising the data on the time grid. This procedure (Delache et al. 1985; Özgüç \& Ataç 1994) maintains the noise characteristic of the time series but destroys all coherent signals, especially those with periods longer than the chosen cut interval of the data. In our time series, we cut the data with a seven day interval. It preserves to some extent the correlation characteristics of the data, so if a period results from a strong correlation inside the data, the number of cases in which it is observed should be rather large. We repeated this simulation 10000 times, every time computing the number of cases in which the recalculated power values 
Table 2. Charactaristic parameters for the minimum original time series (0) and all the next obtained after removing from the original data successively one, two and more sine curves ("-1", "-2", ...) having periods with peaks whose $F A P$ values are smaller than $0.5 \%$ These frequencies are printed in bold letters.

\begin{tabular}{|c|c|c|c|c|c|c|c|c|c|c|c|c|c|c|c|c|c|}
\hline & \multicolumn{2}{|c|}{$\begin{array}{c}\text { The highest } \\
\text { peak at }\end{array}$} & \multirow{2}{*}{\multicolumn{2}{|c|}{$\begin{array}{c}\text { Auto } \\
\text { correlation } \\
\text { lag }\end{array}$}} & \multirow[t]{3}{*}{$k$} & \multirow[t]{3}{*}{$N P$} & \multirow[t]{3}{*}{$\begin{array}{r}F A P \\
{[\%]}\end{array}$} & \multirow{3}{*}{$\begin{array}{c}\% \\
\text { of } \\
\text { var. } \\
\text { exp. }\end{array}$} & & \multicolumn{2}{|c|}{$\begin{array}{c}\text { The highest } \\
\text { peak at }\end{array}$} & \multirow{2}{*}{\multicolumn{2}{|c|}{$\begin{array}{c}\text { Auto } \\
\text { correlation } \\
\text { lag }\end{array}$}} & \multirow[t]{3}{*}{$k$} & \multirow[t]{3}{*}{$N P$} & \multirow[t]{3}{*}{$\begin{array}{r}F A P \\
{[\%]}\end{array}$} & \multirow{3}{*}{$\begin{array}{c}\% \\
\text { of } \\
\text { var. } \\
\text { exp. }\end{array}$} \\
\hline & Freq. & Per. & & & & & & & & Freq. & Per. & & & & & & \\
\hline & {$[\mathrm{nHz}]$} & [day] & 1 & 7 & & & & & & {$[\mathrm{nHz}]$} & [day] & 1 & 7 & & & & \\
\hline 1 & \multicolumn{2}{|c|}{2} & \multicolumn{2}{|c|}{3} & 4 & 5 & 6 & 7 & 1 & & & \multicolumn{2}{|c|}{3} & 4 & 5 & 6 & 7 \\
\hline$I S N$ & & & & & & & & & $M M F$ & & & & & & & & \\
\hline \multirow[t]{3}{*}{0} & $84 a$ & 138 & .839 & .126 & 2.59 & 11.8 & 0.15 & 17.4 & 0 & $858 a$ & 13.5 & .523 & -.187 & 1.56 & 19.0 & 0.00 & 12.6 \\
\hline & & & & & & & & & -1 & $419 a$ & 27.6 & .476 & -.079 & 1.54 & 19.0 & 0.00 & 23.8 \\
\hline & & & & & & & & & -2 & $400 a$ & 28.9 & .390 & -.095 & 1.65 & 13.7 & 0.02 & 30.0 \\
\hline$S V C$ & 405 & & & & & & & & $R R E$ & 405 & & & & & & & \\
\hline 0 & $418 \mathrm{a}$ & 27.7 & .801 & .007 & 2.50 & 19.8 & 0.00 & 21.0 & 0 & $416 a$ & 27.8 & .714 & .014 & 2.25 & 23.4 & 0.00 & 22.6 \\
\hline$S V C$ & 810 & & & & & & & & $R R E$ & 810 & & & & & & & \\
\hline 0 & $423 a$ & 27.4 & .905 & .177 & 1.56 & 49.9 & 0.00 & 35.9 & 0 & $418 a$ & 27.7 & .801 & .218 & 1.46 & 42.9 & 0.00 & 26.4 \\
\hline-1 & $396 a$ & 29.2 & .895 & .285 & 1.71 & 18.7 & 0.00 & 45.2 & -1 & $152 \mathrm{a}$ & 76.2 & .765 & .346 & 1.70 & 27.7 & 0.00 & 40.6 \\
\hline-2 & $150 \mathrm{~b}$ & 77.2 & .883 & .321 & 1.89 & 15.6 & 0.00 & 52.1 & -2 & $381 a$ & 30.4 & .707 & .216 & 2.06 & 15.3 & 0.00 & 48.8 \\
\hline-3 & $76 \mathrm{~b}$ & 152 & .874 & .228 & 2.01 & 14.1 & 0.01 & 57.7 & -3 & $268 \mathrm{~b}$ & 43.2 & .667 & .242 & 2.07 & 11.8 & 0.14 & 54.2 \\
\hline-4 & $529 \mathrm{~b}$ & 21.9 & .852 & .105 & 2.10 & 11.2 & 0.28 & 61.9 & & & & & & & & & \\
\hline-5 & $238 \mathrm{~b}$ & 48.6 & .836 & .164 & 2.11 & 11.5 & 0.20 & 66.0 & & & & & & & & & \\
\hline$S V C$ & 1215 & & & & & & & & $R R E$ & 1215 & & & & & & & \\
\hline 0 & $424 a$ & 27.3 & .912 & .219 & 1.60 & 34.6 & 0.00 & 26.7 & 0 & $157 \mathrm{a}$ & 73.7 & .757 & .271 & 1.72 & 18.8 & 0.00 & 13.7 \\
\hline-1 & $80 \mathrm{~b}$ & 145 & .905 & .297 & 1.72 & 14.8 & 0.01 & 34.5 & -1 & $420 a$ & 27.6 & .718 & .160 & 1.91 & 18.7 & 0.00 & 26.7 \\
\hline-2 & $151 \mathrm{~b}$ & 76.6 & .893 & .207 & 1.79 & 15.0 & 0.01 & 41.8 & -2 & $383 \mathrm{~b}$ & 30.2 & .683 & 221 & 1.93 & 12.2 & 0.10 & 34.3 \\
\hline-3 & $528 \mathrm{~b}$ & 21.9 & .886 & .112 & 1.90 & 12.1 & 0.11 & 47.5 & -3 & $267 \mathrm{~b}$ & 43.4 & .649 & .233 & 1.89 & 11.8 & 0.14 & 40.6 \\
\hline-4 & $113 \mathrm{~b}$ & 102 & .873 & .176 & 1.94 & 11.5 & 0.20 & 52.9 & & & & & & & & & \\
\hline-5 & $498 \mathrm{~b}$ & 23.2 & .855 & .084 & 1.95 & 11.8 & 0.15 & 57.8 & & & & & & & & & \\
\hline-6 & $242 b$ & 47.8 & .839 & .129 & 2.18 & 11.8 & 0.15 & 62.5 & & & & & & & & & \\
\hline$S V C$ & 1620 & & & & & & & & $R R E$ & 1620 & & & & & & & \\
\hline \multirow[t]{3}{*}{0} & $425 a$ & 27.2 & .898 & .111 & 2.15 & 23.1 & 0.00 & 25.0 & 0 & $155 \mathrm{~b}$ & 74.7 & .687 & 197 & 2.00 & 11.7 & 0.16 & 10.6 \\
\hline & & & & & & & & & -1 & $420 \mathrm{~b}$ & 27.6 & .653 & .123 & 2.07 & 11.2 & 0.28 & 19.3 \\
\hline & & & & & & & & & -2 & $382 \mathrm{~b}$ & 30.3 & .627 & .159 & 2.12 & 10.8 & 0.40 & 27.2 \\
\hline$S V C$ & 2800 & & & & & & & & $R R E$ & 2800 & & & & & & & \\
\hline 0 & $85 b$ & 136 & .931 & .134 & 2.38 & 13.0 & 0.05 & 17.4 & 0 & $383 \mathrm{~b}$ & 30.2 & .647 & .044 & 2.32 & 7.8 & 7.78 & 7.6 \\
\hline-1 & $424 a$ & 27.3 & .921 & .020 & 2.57 & 13.5 & 0.03 & 28.0 & & & & & & & & & \\
\hline$S V C$ & 4995 & & & & & & & & $R R E$ & 4995 & & & & & & & \\
\hline 0 & $90 a$ & 129 & .862 & .286 & 2.00 & 18.3 & 0.00 & 14.3 & 0 & $53 a$ & 218 & .710 & .347 & 1.64 & 23.5 & 0.00 & 19.4 \\
\hline-1 & $124 a$ & 93.3 & .832 & .166 & 2.20 & 13.9 & 0.02 & 24.6 & -1 & $158 \mathrm{a}$ & 73.2 & .625 & .224 & 1.83 & 19.5 & 0.00 & 30.3 \\
\hline$S V C$ & 8800 & & & & & & & & $R R E$ & 8800 & & & & & & & \\
\hline 0 & $122 \mathrm{~b}$ & 94.9 & .635 & .355 & 1.63 & 15.8 & 0.00 & 10.3 & 0 & $156 \mathrm{~b}$ & 74.2 & .638 & .396 & 1.52 & 16.2 & 0.00 & 10.0 \\
\hline \multirow[t]{2}{*}{-1} & $154 \mathrm{~b}$ & 75.2 & .593 & .289 & 1.71 & 14.9 & 0.01 & 19.9 & -1 & $121 \mathrm{~b}$ & 95.6 & .598 & .339 & 1.54 & 14.3 & 0.01 & 18.1 \\
\hline & & & & & & & & & -2 & $83 \mathrm{~b}$ & 139 & .558 & .285 & 1.55 & 11.8 & 0.15 & 24.3 \\
\hline
\end{tabular}

The columns of the table show: 1: time series ("-1" indicates that the one sine curve with period given a row above was removed from the original data, "-2" indicates that the two sine curves with periods given in two rows above were removed from the original data, and so on), 2: frequency and period of the highest peak in a given time series, a small letter following a frequency value indicates to which interval of probability ("a": $<0.1 \%$, "b": $0.1-1 \%$, "c": $1-5 \%$ ) this period belongs to after the randomising procedure, 3: the autocorrelation coefficients calculated with two lags equal 1 and 7 days respectively, 4: the normalization factor $k$ computed according to the procedure described in Sect. 3, 5: the normalized power equal to the Scargle power divided by $k, 6$ : the $F A P$ value resulting from the normalized power, 7 : in successive rows a percentage of the original data variance explained by the prominent sinusoidal signals found in a given type of data.

for the periods having peaks in the original spectrum are equal to or larger than the peaks power of the real data. The results of these calculations are presented in Tables 4 and 5 as small letters situated after the frequency of the peaks found in the original periodograms. The letter "a" indicates that, for 10000 simulations, in less then 10 cases the peak value at the given frequency exceeded the corresponding peak power of the real data. Successive letters mark the intervals for which the probability (calculated from 10000 simulations) of obtaining as high a peak as in the original periodogram by chance are as follows: "b": $0.1-1 \%$, "c": $1-5 \%$, "d": 5-15\%.
When more than one periodic signal is present in the data, multiple significant peaks appear in the periodogram. Alternatively, a true signal at frequency $\omega_{\circ}$ can cause peaks in the periodogram at frequencies other than $\omega_{\circ}$ because of the finite length of the data and irregularities in the data spacing. A useful procedure for determining whether any additional peaks with significant false alarm probability are physically real is an iterative peak removal technique (Delache \& Scherrer 1983; Horne \& Baliunas 1986). The highest peak in the original data periodogram provides the frequency $\omega_{\circ}$ corresponding to the strongest sinusoidal signal present in the data. Using 
Table 3. Same as Table 2 but for the rising phase data.

\begin{tabular}{|c|c|c|c|c|c|c|c|c|c|c|c|c|c|c|c|c|c|}
\hline & \multicolumn{2}{|c|}{$\begin{array}{c}\text { The highest } \\
\text { peak at }\end{array}$} & \multirow{2}{*}{\multicolumn{2}{|c|}{$\begin{array}{c}\text { Auto } \\
\text { correlation } \\
\text { lag }\end{array}$}} & \multirow[t]{3}{*}{$k$} & \multirow[t]{3}{*}{$N P$} & \multirow[t]{3}{*}{$\begin{array}{r}F A P \\
{[\%]}\end{array}$} & \multirow{4}{*}{$\begin{array}{c}\% \\
\text { of } \\
\text { var. } \\
\text { exp. } \\
7\end{array}$} & & \multicolumn{2}{|c|}{$\begin{array}{c}\text { The highest } \\
\text { peak at }\end{array}$} & \multirow{2}{*}{\multicolumn{2}{|c|}{$\begin{array}{c}\text { Auto } \\
\text { correlation } \\
\text { lag }\end{array}$}} & \multirow[t]{3}{*}{$k$} & $N P$ & $\begin{array}{r}F A P \\
{[\%]}\end{array}$ & $\begin{array}{l}\% \\
\text { of }\end{array}$ \\
\hline & Freq. & Per. & & & & & & & & Freq. & Per. & & & & & & var. \\
\hline & {$[\mathrm{nHz}]$} & [day] & 1 & 7 & & & & & & {$[\mathrm{nHz}]$} & [day] & 1 & 7 & & & & exp. \\
\hline 1 & & & & & 4 & 5 & 6 & & 1 & & & & & 4 & 5 & 6 & 7 \\
\hline$I S N$ & & & & & & & & & $M M F$ & & & & & & & & \\
\hline 0 & $442 \mathrm{a}$ & 26.2 & .906 & .150 & 2.19 & 19.2 & 0.00 & 44.6 & 0 & $799 a$ & 14.5 & .650 & -.415 & 1.54 & 25.8 & 0.00 & 15.5 \\
\hline-1 & $127 a$ & 91.1 & .897 & .179 & 2.37 & 13.9 & 0.02 & 49.7 & -1 & $844 a$ & 13.7 & .634 & -.340 & 1.70 & 26.9 & 0.00 & 30.1 \\
\hline & & & & & & & & & -2 & $876 a$ & 13.2 & .548 & -.187 & 1.93 & 12.2 & 0.14 & 36.4 \\
\hline$S V C$ & 405 & & & & & & & & $R R E$ & 405 & & & & & & & \\
\hline 0 & $418 \mathrm{a}$ & 27.7 & .731 & .086 & 1.90 & 24.5 & 0.00 & 21.9 & 0 & $418 a$ & 27.7 & .641 & .064 & 2.15 & 15.2 & 0.01 & 9.8 \\
\hline-1 & $55 a$ & 210 & .695 & .102 & 2.10 & 14.3 & 0.02 & 28.8 & -1 & $52 a$ & 223 & .609 & .072 & 2.23 & 13.2 & 0.05 & 17.9 \\
\hline-2 & $444 a$ & 26.1 & .663 & .017 & 2.15 & 11.1 & 0.39 & 33.9 & & & & & & & & & \\
\hline$S V C$ & 810 & & & & & & & & $R R E$ & 810 & & & & & & & \\
\hline 0 & $443 a$ & 26.1 & .935 & .215 & 1.01 & 74.0 & 0.00 & 33.0 & 0 & $417 \mathrm{a}$ & 27.8 & .863 & .206 & 1.30 & 52.0 & 0.00 & 20.2 \\
\hline-1 & $416 a$ & 27.8 & .924 & .300 & 1.21 & 50.4 & 0.00 & 45.4 & -1 & $450 a$ & 25.7 & .842 & .275 & 1.58 & 27.2 & 0.00 & 30.2 \\
\hline-2 & $75 a$ & 154 & .918 & .385 & 1.32 & 38.2 & 0.00 & 53.5 & -2 & $56 a$ & 207 & .824 & .323 & 1.74 & 20.3 & 0.00 & 37.4 \\
\hline-3 & $456 a$ & 25.4 & .904 & .275 & 1.51 & 20.1 & 0.00 & 58.0 & -3 & $71 b$ & 163 & .806 & .245 & 1.91 & 13.2 & 0.05 & 42.0 \\
\hline-4 & $62 a$ & 187 & .893 & .318 & 1.59 & 21.5 & 0.00 & 62.3 & & & & & & & & & \\
\hline-5 & $103 a$ & 112 & .878 & .239 & 1.67 & 21.4 & 0.00 & 66.4 & & & & & & & & & \\
\hline-6 & $477 a$ & 24.3 & .861 & .147 & 1.80 & 14.2 & 0.02 & 69.0 & & & & & & & & & \\
\hline-7 & $385 a$ & 30.1 & .853 & .193 & 1.74 & 15.4 & 0.01 & 71.5 & & & & & & & & & \\
\hline-8 & $127 \mathrm{~b}$ & 91.1 & .843 & .205 & 1.76 & 15.2 & 0.01 & 73.9 & & & & & & & & & \\
\hline-9 & $562 b$ & 20.6 & .829 & .143 & 1.77 & 12.8 & 0.07 & 75.6 & & & & & & & & & \\
\hline-10 & $498 b$ & 23.2 & .819 & .184 & 1.84 & 12.3 & 0.12 & 77.3 & & & & & & & & & \\
\hline$S V C$ & 1215 & & & & & & & & $R R E$ & 1215 & & & & & & & \\
\hline 0 & $444 a$ & 26.1 & .957 & .240 & 1.14 & 68.5 & 0.00 & 36.4 & 0 & $418 a$ & 27.7 & .872 & .253 & 1.37 & 33.4 & 0.00 & 14.4 \\
\hline-1 & $77 a$ & 150 & .948 & .333 & 1.41 & 38.7 & 0.00 & 46.6 & -1 & $449 a$ & 25.8 & .861 & .303 & 1.52 & 27.6 & 0.00 & 24.9 \\
\hline-2 & $416 a$ & 27.8 & .938 & .206 & 1.61 & 35.2 & 0.00 & 55.6 & -2 & $76 a$ & 152 & .845 & .349 & 1.74 & 24.9 & 0.00 & 34.3 \\
\hline-3 & $104 a$ & 111 & .935 & .261 & 1.74 & 21.8 & 0.00 & 60.6 & -3 & $59 a$ & 196 & .823 & .261 & 1.88 & 15.7 & 0.00 & 40.0 \\
\hline-4 & $457 a$ & 25.3 & .922 & .159 & 1.93 & 14.3 & 0.02 & 64.1 & & & & & & & & & \\
\hline-5 & $64 \mathrm{~b}$ & 181 & .912 & .188 & 1.93 & 14.7 & 0.01 & 67.4 & & & & & & & & & \\
\hline-6 & $129 b$ & 89.7 & .900 & .109 & 2.00 & 14.3 & 0.02 & 70.3 & & & & & & & & & \\
\hline-7 & $480 a$ & 24.1 & .893 & .035 & 1.94 & 15.2 & 0.01 & 73.0 & & & & & & & & & \\
\hline-8 & $563 b$ & 20.6 & .888 & .077 & 1.96 & 12.7 & 0.08 & 75.0 & & & & & & & & & \\
\hline-9 & $499 \mathrm{~b}$ & 23.2 & .882 & .118 & 2.02 & 13.2 & 0.05 & 77.0 & & & & & & & & & \\
\hline-10 & $385 b$ & 30.1 & .874 & .158 & 2.04 & 11.9 & 0.18 & 78.7 & & & & & & & & & \\
\hline-11 & $49 b$ & 236 & .869 & .161 & 2.03 & 10.2 & 0.31 & 80.3 & & & & & & & & & \\
\hline-12 & $361 b$ & 32.1 & .858 & .116 & 1.92 & 12.6 & 0.09 & 81.9 & & & & & & & & & \\
\hline-13 & $148 b$ & 78.2 & .850 & .128 & 1.84 & 12.5 & 0.10 & 83.1 & & & & & & & & & \\
\hline$S V C$ & 1620 & & & & & & & & $R R E$ & 1620 & & & & & & & \\
\hline 0 & $444 a$ & 26.1 & .955 & .250 & 1.44 & 47.7 & 0.00 & 38.6 & 0 & $419 a$ & 27.6 & .831 & .149 & 1.89 & 21.8 & 0.00 & 12.2 \\
\hline-1 & $77 a$ & 150 & .946 & .329 & 1.68 & 33.6 & 0.00 & 48.9 & -1 & $78 a$ & 148 & .816 & .181 & 2.03 & 22.6 & 0.00 & 23.8 \\
\hline-2 & $416 a$ & 27.8 & .934 & .196 & 1.93 & 25.0 & 0.00 & 56.3 & -2 & $450 a$ & 25.7 & .787 & .061 & 2.26 & 16.1 & 0.00 & 31.9 \\
\hline-3 & $128 \mathrm{a}$ & 90.4 & .930 & .239 & 2.03 & 17.8 & 0.00 & 61.0 & & & & & & & & & \\
\hline-4 & $105 b$ & 110 & .920 & .064 & 2.17 & 12.3 & 0.12 & 64.2 & & & & & & & & & \\
\hline$S V C$ & 2800 & & & & & & & & $R R E$ & 2800 & & & & & & & \\
\hline 0 & $443 a$ & 26.1 & .927 & .251 & 1.47 & 41.0 & 0.00 & 37.9 & 0 & $78 a$ & 148 & .775 & .178 & 1.94 & 21.5 & 0.00 & 11.8 \\
\hline-1 & $77 a$ & 150 & .917 & .315 & 1.65 & 34.3 & 0.00 & 47.8 & -1 & $421 a$ & 27.5 & .744 & .066 & 2.13 & 16.7 & 0.00 & 20.7 \\
\hline-2 & $416 a$ & 27.8 & .900 & .180 & 1.93 & 19.2 & 0.00 & 53.5 & & & & & & & & & \\
\hline-3 & $128 b$ & 90.4 & .893 & .207 & 2.09 & 13.4 & 0.04 & 57.2 & & & & & & & & & \\
\hline-4 & $430 \mathrm{~b}$ & 26.9 & .883 & .146 & 2.08 & 12.2 & 0.12 & 60.6 & & & & & & & & & \\
\hline-5 & $487 a$ & 23.8 & .876 & .159 & 1.97 & 15.2 & 0.01 & 64.1 & & & & & & & & & \\
\hline-6 & $66 \mathrm{~b}$ & 175 & .868 & .200 & 2.07 & 14.0 & 0.02 & 67.7 & & & & & & & & & \\
\hline-7 & $52 a$ & 223 & .854 & .112 & 2.16 & 15.6 & 0.00 & 71.2 & & & & & & & & & \\
\hline$S V C$ & 4995 & & & & & & & & $R R E$ & 4995 & & & & & & & \\
\hline 0 & $442 \mathrm{a}$ & 26.2 & .891 & .182 & 1.75 & 31.6 & 0.00 & 37.5 & 0 & $79 \mathrm{a}$ & 147 & .755 & .009 & 2.66 & 9.1 & 2.78 & 7.1 \\
\hline-1 & $77 a$ & 150 & .870 & .221 & 2.00 & 21.3 & 0.00 & 45.3 & & & & & & & & & \\
\hline-2 & $420 \mathrm{~b}$ & 27.6 & .859 & .114 & 2.20 & 11.3 & 0.33 & 49.4 & & & & & & & & & \\
\hline-3 & $103 b$ & 112 & .856 & .132 & 2.34 & 11.0 & 0.39 & 53.4 & & & & & & & & & \\
\hline$S V C$ & 8800 & & & & & & & & $R E$ & 8800 & & & & & & & \\
\hline 0 & $79 a$ & 147 & .756 & .365 & 1.74 & 42.7 & 0.00 & 26.9 & 0 & $81 a$ & 143 & .726 & .338 & 1.89 & 33.5 & 0.00 & 19.6 \\
\hline-1 & $66 a$ & 175 & .696 & .189 & 2.20 & 25.9 & 0.00 & 40.5 & -1 & $67 a$ & 173 & .668 & .185 & 2.28 & 20.9 & 0.00 & 31.5 \\
\hline & & & & & & & & & -2 & $51 a$ & 227 & .617 & .073 & 2.55 & 11.3 & 0.32 & 37.8 \\
\hline
\end{tabular}

the method of least squares the phase and amplitude of this sinusoidal signal are fitted from the original data. This allows subtraction of the best fitted sine curve from the time series and then recalculation of a new periodogram. This procedure is repeated as long as the FAP of the peaks connected with subtracted sine curves are smaller than $0.5 \%$, producing the main periods in each of the analysed time series. To see the effect of removing these peaks from the original data, we present in Fig. 3 the six consecutive periodograms, calculated according to this procedure for the case of the minimum $S V C 810$ time series. We see that the original periodogram is dominated by a strong peak at frequency $423 \mathrm{nHz}\left(27^{\mathrm{d}} 4\right)$, so the structure of the periodogram near this frequency is difficult to 
recognise. When the sine curve with this period is removed from the data, the new period at 29.2 begins to be visible (Fig. 3b). Subtracting the next sine curves from the data we come to Fig. 3f which presents the periodogram of the last descendant time series having period whose peak value gives $F A P$ smaller than $0.5 \%$. Comparing all the periodograms seen in this figure, we can conclude that the period values indicated by peak positions do not change much during the removed procedure, which confirms the significance of the periods found. The results of the removal procedure are summarised in Table 2 for the minimum and in Table 3 for the rising phase. Both tables show for the original time series (marked by 0 ), and those with successively removed sine curves (denoted by $-1,-2,-3 .$.$) such characteristic parameters as: fre-$ quency and period of the highest peak, the autocorrelation coefficients, the normalization factor $k$, the normalized power equal to the Scargle power divided by $k$, the $F A P$ value resulting from the normalized power.

\section{Results and discussion}

Most of the calculated periodograms have been obtained from radio observations ( $S V C$ and $R R E$ time series calculated for seven different frequencies), but the $I S N$ and $M M F$ data were also analysed. The final periodograms obtained when all the main sinusoidal signals given in Tables 2 and 3 are subtracted from the original data are presented in Figs. 4-7. Careful examination of these periodograms shows that many periods having a formal significance level near 30\% are seen in time series coming from different observational data. This fact allow us to suggest that even those periods could be real.

Taking into account all the information about the periods found obtained with the three different approaches to the data described in Sect.3, we prepare Table 4 for the minimum, and Table 5 for the rising phase, which bring together information about characteristic periodicities (lines) observed in these phases of the solar cycle 23 (we use the spectroscopic term "line" for the mean period calculated from the periods observed in various time series, which, as we suppose, represent the same characteristic periodicity). As two time series were created from the radio data at each frequency, we use two successive rows in the radio data columns to separate periods observed in $S V C$ and $R R E$ radio time series. The exact frequencies of the detected periods were found with a $1 \mathrm{nHz}$ resolution in the vicinity of peaks seen in periodograms constructed from normalized power values on the grid of the independent frequencies appropriate to the given window. In all the figures, the points marked in the presented periodograms are situated in the places resulting from the grid of the independent frequencies, while the solid lines are drawn from the power values computed every $1 \mathrm{nHz}$. To discribe in some measure a "strength" of the line we introduced so called the importance number of the line. This number shows how large is support for this line from all the analysed time series. It is calculated according to the following rule. The each letter "a" in the line description columns (Cols. 12-14) gives for the importance number " 5 ", the letter "b" gives " 3 " , and the letter "c" only "1". It is only one line with the importance number " 5 " in Tables 4 and 5 . There are a few lines with a smaller importance number than " 5 " recognised in the analysed data, but we do not include them in Tables 4 and 5. However, it is important to notice that some of the lines included in Tables 4 and 5 can be unreal in a sense that they are created from periods which in fact belong to two different but neighboring lines. For such a case the calculated mean period is somewhere between the periods of these two neighboring, unknown lines. The probability of such a situation increses for lines having long periods and large values of $\Delta f$ (Col. 10, second row).

To aid in further discussion of the lines we have prepared Fig. 8, which shows all the lines present in Tables 4 and 5. The level of darkness in this 3D graph illustrate the importance number of the lines. A close look at Fig. 8 shows a clear difference among the 18 lines observed in the minimum and the 22 lines found in the rising phase of solar sunspot activity cycle. Although the 9 lines have almost the same periods in both the phases, the strength (measured indirectly by the importance number) of nearly all the 18 lines change, indicating that perhaps the physical mechanisms responsible for them also change with the solar phase. From Fig. 8 it is evident that the lines in the rising phase gather into three groups:

1. Magnetic lines: all lines with periods shorter than 16 days. They have small importance numbers and were included on our list only because of very high peaks observed at these short periods in the $M M F$ (mean magnetic field) time series. In the minimum, only one line (13.5) from this group is enough strong to be present in Fig. 8, but in the minimum $M M F$ periodogram (see Fig. 6) some of them are easily seen;

2. Rotational lines: all lines with periods inside the 2534 days time interval of the Sun's rotation. The three lines from this group are visible in the minimum. The line 27.3 has the largest importance number and results probably from the rotation of such solar phenomena as new solar cycle sunspots as well as the long-lived coronal streamer structure observed during the 1996 minimum (Lewis et al. 1999). In the rising phase the period of this line shifts to 27.7 . The next two lines 29.1 and 30.3 are stronger in the minimum than in the rising phase. This additionally supports the supposition that they are associated with some medium, and large scale magnetic structures (coronal neutral sheet, global neutral lines) which dominate in the minimum and then decline (Lantos et al. 1992; Lantos 1999). In the rising phase four new lines appear $(25.2,26$ d 0 , $26.8,31.8)$. Three have periods shorter than those discussed above. One of them, having a period of $26 \mathrm{~d} 0$, is the third dominant line of this phase. We suppose that this line is strongly connected with eruption of new active-region magnetic fields emerging within the 

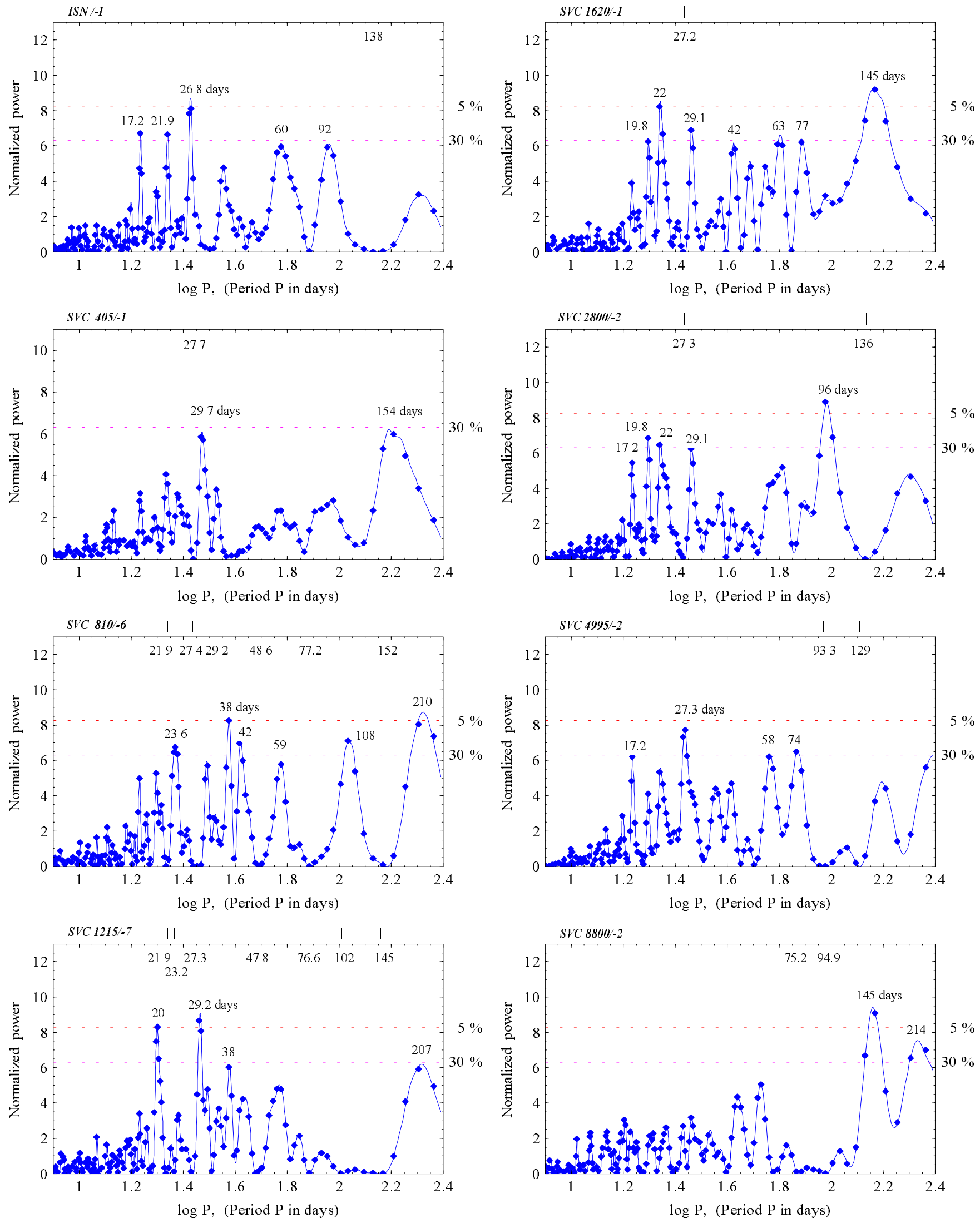

Fig. 4. The normalised periodograms of the minimum $I S N$ and $S V C$ time series after removing from the original data all the sine curves having periods with $F A P$ values smaller than $0.5 \%$. The removed periods are shown at the top of each graph. The dashed lines indicate $F A P$ significance levels. 

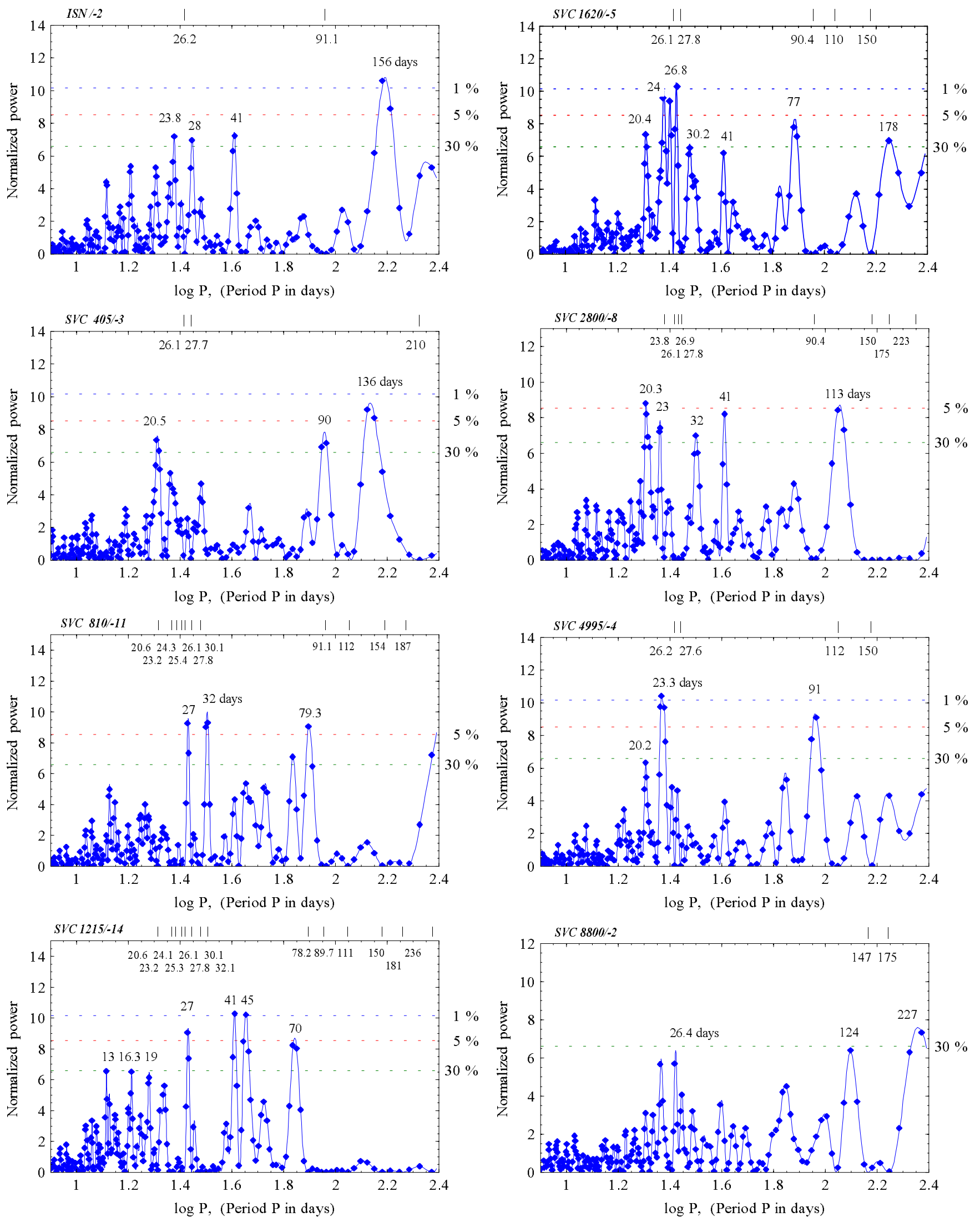

Fig. 5. Same as Fig. 4. but for the rising phase $I S N$ and $S V C$ time series after removing from the original data all the sine curves having periods with $F A P$ values smaller than $0.5 \%$. 

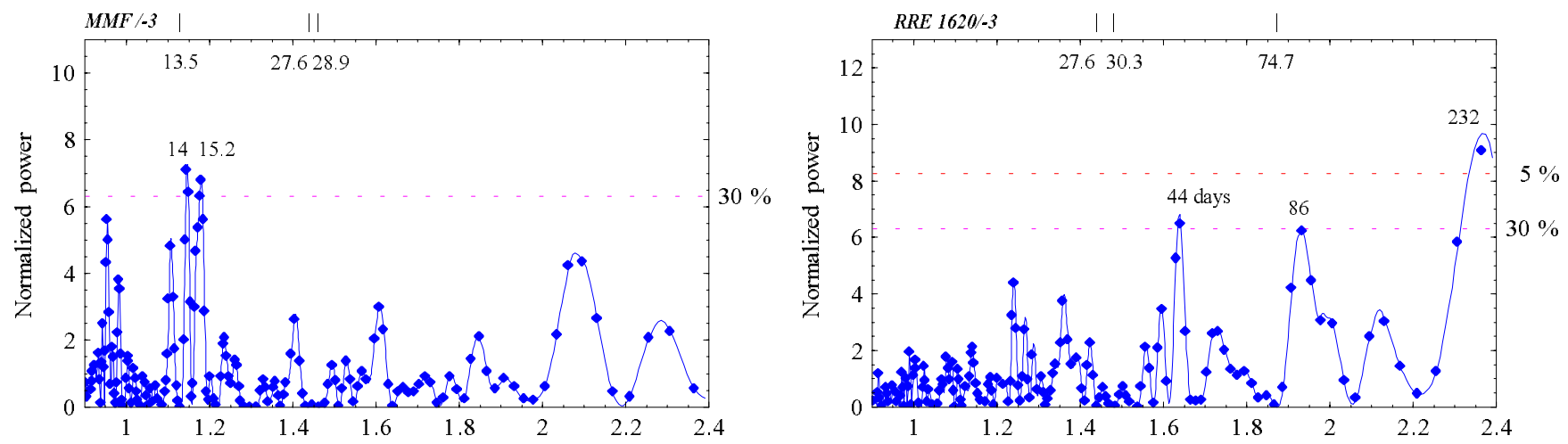

$\log \mathrm{P}$, (Period $\mathrm{P}$ in days)
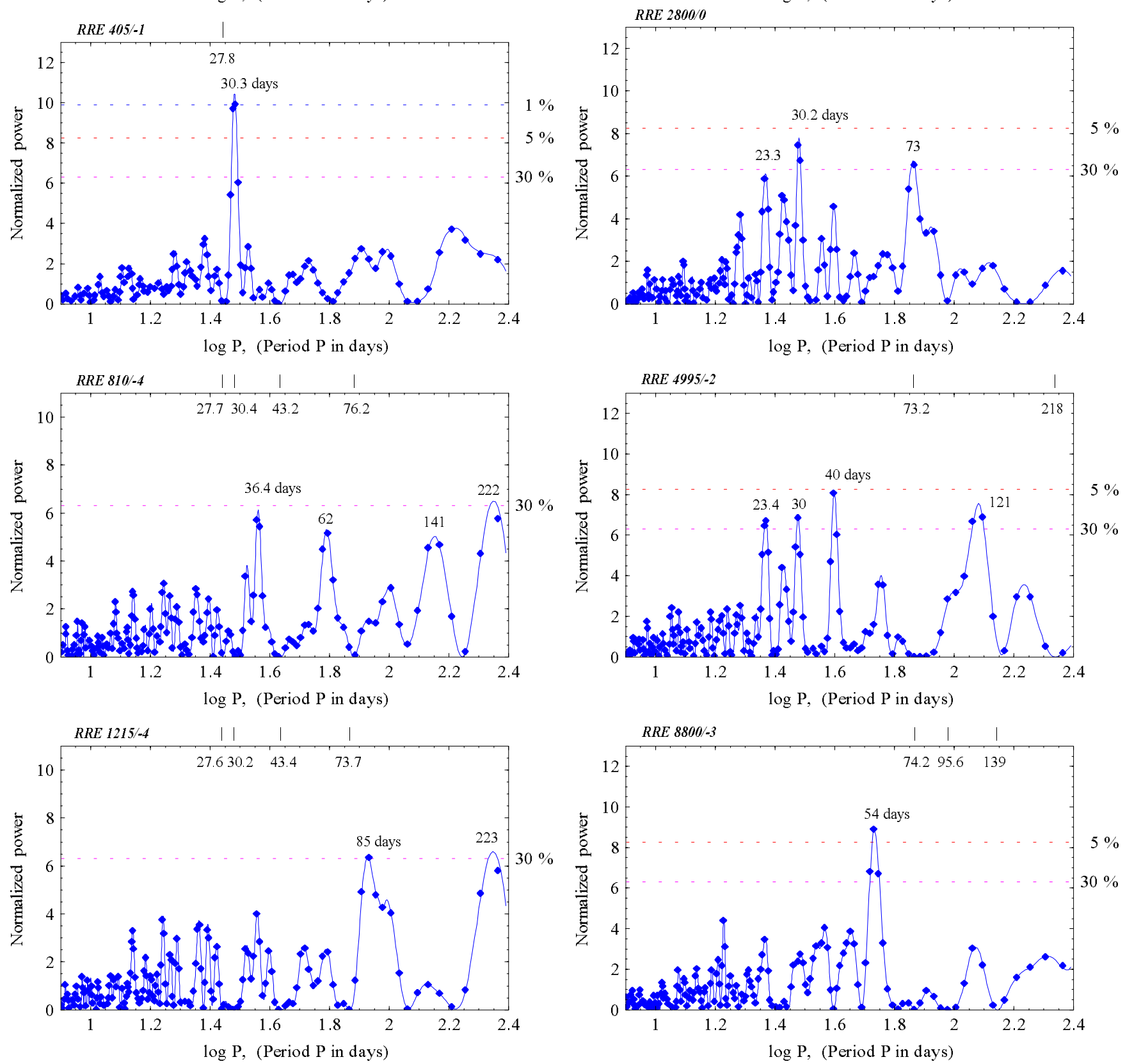

Fig. 6. Same as Fig. 4. but for the minimum $M M F$ and $R R E$ time series after removing from the original data all the sine curves having periods with $F A P$ values smaller than $0.5 \%$. 

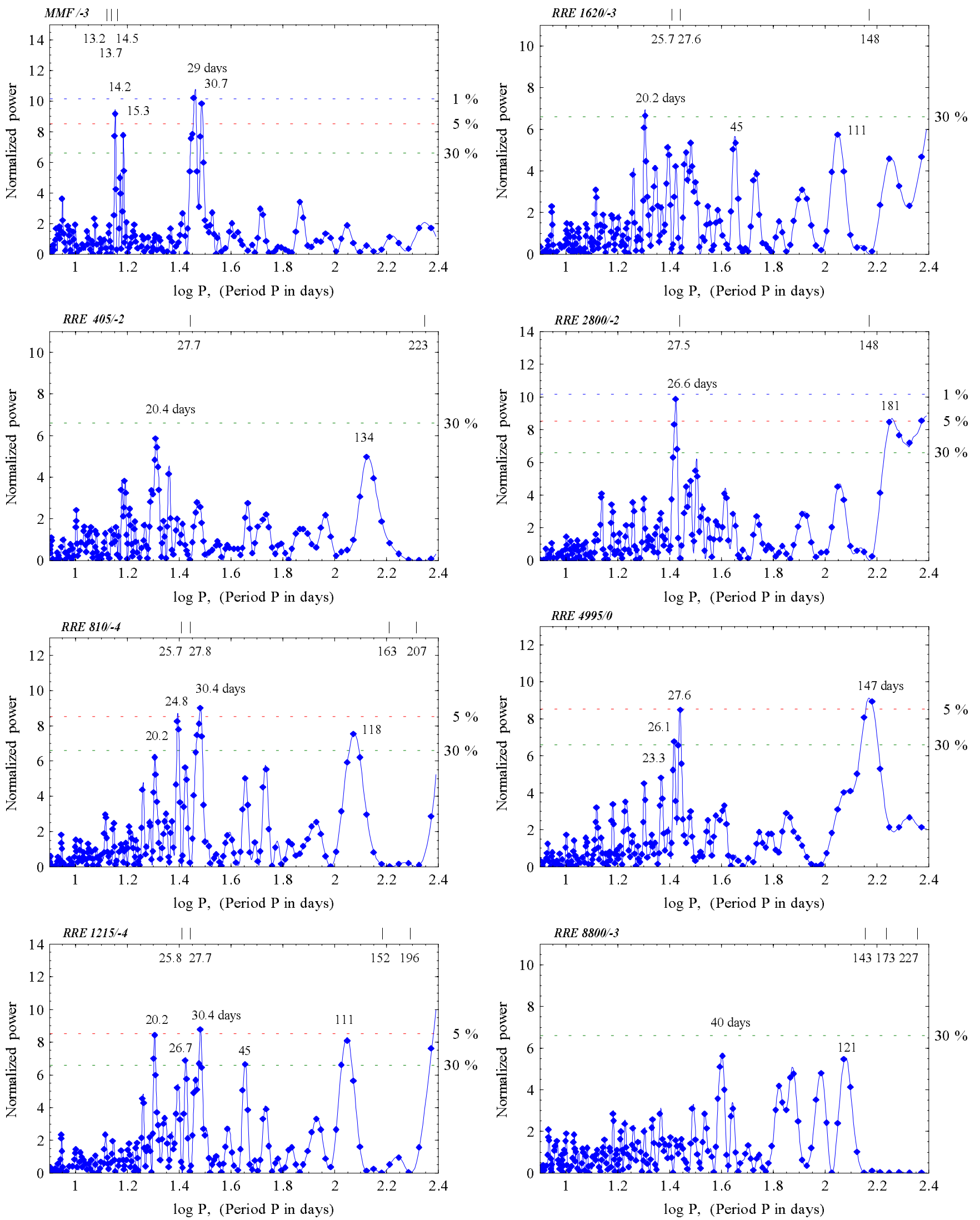

Fig. 7. Same as Fig. 4. but for the rising phase $M M F$ and $R R E$ time series after removing from the original data all the sine curves having periods with $F A P$ values smaller than $0.5 \%$. 
Table 4. The characteristic periodicities (lines) observed in the minimum.

\begin{tabular}{|c|c|c|c|c|c|c|c|c|c|c|c|c|c|c|c|}
\hline \multirow[t]{3}{*}{$M M F$} & \multirow{3}{*}{$\begin{array}{c}S V C \\
R R E \\
405\end{array}$} & \multirow{3}{*}{$\begin{array}{c}S V C \\
R R E \\
810\end{array}$} & \multirow{2}{*}{$\begin{array}{l}S V C \\
R R E\end{array}$} & \multirow{2}{*}{$\begin{array}{l}S V C \\
R R E\end{array}$} & \multirow{2}{*}{$\begin{array}{l}S V C \\
R R E\end{array}$} & \multirow{2}{*}{$\begin{array}{l}S V C \\
R R E\end{array}$} & \multirow{3}{*}{$\begin{array}{l}S V C \\
R R E \\
8800\end{array}$} & \multirow[t]{3}{*}{$I S N$} & \multicolumn{2}{|c|}{ Line } & \multirow[t]{3}{*}{$M$} & \multirow{3}{*}{$\begin{array}{l}S V C \\
R R E\end{array}$} & \multirow{3}{*}{$\begin{array}{l}I S N \\
\text { Imp. }\end{array}$} & \multicolumn{2}{|c|}{ Time series } \\
\hline & & & & & & & & & {$[\mathrm{nHz}]$} & [day] & & & & $N P$ & $F A P$ \\
\hline & & & 1215 & 1620 & 2800 & 4995 & & & $\Delta f$ & & & & & & {$[\%]$} \\
\hline 1 & 2 & 3 & 4 & 5 & 6 & 7 & 8 & 9 & 10 & 11 & 12 & 13 & 14 & 15 & 16 \\
\hline \multirow{8}{*}{$858 a$} & $851 d$ & & & & & & & & 13 & & & + & 6 & 19.0 & 0.00 \\
\hline & $673 \mathrm{c}$ & $679 \mathrm{c}$ & $679 \mathrm{~d}$ & $675 \mathrm{c}$ & $674 \mathrm{~b}$ & $672 \mathrm{~b}$ & & $672 \mathrm{~b}$ & 673.0 & 17.2 & - & $2 \mathrm{~b} 3 \mathrm{c}$ & $\mathrm{b}$ & $I S$ & $\mathrm{~N} /-1$ \\
\hline & & & $662 \mathrm{c}$ & $665 c$ & & & $685 \mathrm{c}$ & & 23 & & & $3 \mathrm{c}$ & 15 & 6.7 & 20.93 \\
\hline & $588 \mathrm{~d}$ & $585 \mathrm{c}$ & $580 \mathrm{~b}$ & $584 \mathrm{c}$ & $584 \mathrm{~b}$ & $585 \mathrm{~d}$ & & $583 \mathrm{c}$ & 586.0 & 19.8 & - & $2 \mathrm{~b} 2 \mathrm{c}$ & $\mathrm{c}$ & $S V C$ & $1215 /-7$ \\
\hline & $534 \mathrm{~b}$ & $529 b$ & $528 \mathrm{~b}$ & $526 \mathrm{~b}$ & $526 \mathrm{~b}$ & $525 \mathrm{c}$ & & $529 \mathrm{~b}$ & 528.1 & 21.9 & - & $5 \mathrm{bc}$ & $\mathrm{b}$ & $S V C$ & $1215 /-3$ \\
\hline & $548 \mathrm{~d}$ & & & & & & & & 23 & & & + & 19 & 12.1 & 0.11 \\
\hline & & $491 \mathrm{c}$ & $498 \mathrm{~b}$ & & & & & & 496.8 & 23.3 & - & $\mathrm{bc}$ & $\overline{-}$ & $\overline{S V C}$ & $1215 /-5$ \\
\hline & & $512 \mathrm{~d}$ & $503 d$ & $504 \mathrm{c}$ & $496 \mathrm{~b}$ & $495 \mathrm{~b}$ & $497 d$ & & 21 & & & $2 \mathrm{bc}$ & 11 & 11.8 & 0.15 \\
\hline \multirow[t]{2}{*}{$419 a$} & $418 a$ & $423 a$ & $424 a$ & $425 a$ & $424 a$ & $424 \mathrm{~b}$ & & $432 \mathrm{a}$ & 423.5 & 27.3 & $\mathrm{a}$ & $5 \mathrm{ab}$ & $\bar{a}$ & $S V C$ & $810 / 0$ \\
\hline & $416 a$ & $418 \mathrm{a}$ & $420 a$ & $420 b$ & $434 \mathrm{c}$ & $432 \mathrm{c}$ & & & 18 & & & $3 a b 2 c$ & 58 & 49.9 & 0.00 \\
\hline \multirow[t]{5}{*}{$400 a$} & & $396 a$ & $397 \mathrm{~b}$ & $398 \mathrm{c}$ & $398 \mathrm{c}$ & & & & 397.8 & 29.1 & $\mathrm{a}$ & $\mathrm{ab2c}$ & - & $S V C$ & $810 /-1$ \\
\hline & & & & & & & & & 4 & & & - & 15 & 18.7 & 0.00 \\
\hline & $382 \mathrm{a}$ & $381 \mathrm{a}$ & $383 \mathrm{~b}$ & $382 b$ & $383 \mathrm{~b}$ & $386 \mathrm{~b}$ & & & 18 & & & $2 \mathrm{a} 4 \mathrm{~b}$ & 28 & 15.3 & 0.00 \\
\hline & & $307 \mathrm{c}$ & $306 c$ & & $308 \mathrm{~d}$ & & & & 299.5 & 39 & - & $2 \mathrm{c}$ & - & $S V C$ & $810 /-6$ \\
\hline & & & & $295 \mathrm{~d}$ & $293 \mathrm{c}$ & $292 b$ & & & 16 & & & bc & 6 & 8.2 & 5.14 \\
\hline \multirow[t]{12}{*}{$286 \mathrm{~d}$} & & $272 \mathrm{c}$ & $265 \mathrm{~d}$ & $275 \mathrm{c}$ & & $281 d$ & $265 d$ & & 270.6 & 43 & + & $2 \mathrm{c}$ & - & $R R E$ & $810 /-3$ \\
\hline & & $268 b$ & $267 \mathrm{~b}$ & $266 \mathrm{~b}$ & & & & & 21 & & & $3 b$ & 11 & 11.8 & 0.14 \\
\hline & & $238 b$ & $242 b$ & $238 \mathrm{~d}$ & & & & & 240.0 & 48 & - & $2 \mathrm{~b}$ & - & $S V C$ & $1215 /-6$ \\
\hline & & & & & & & & & 4 & & & - & 6 & 11.8 & 0.14 \\
\hline & $197 d$ & $195 \mathrm{c}$ & $197 \mathrm{c}$ & & $195 \mathrm{c}$ & $198 \mathrm{c}$ & $217 d$ & $194 \mathrm{c}$ & 199.0 & 58 & - & $4 \mathrm{c}$ & $\mathrm{c}$ & $R R E$ & $8800 /-3$ \\
\hline & & & & $217 \mathrm{~d}$ & & $204 d$ & $215 \mathrm{c}$ & & 23 & & & $\mathrm{c}$ & 6 & 8.9 & 2.55 \\
\hline & & $150 \mathrm{~b}$ & $151 b$ & $150 \mathrm{c}$ & $147 \mathrm{~d}$ & $156 \mathrm{c}$ & $154 b$ & & 154.4 & 75 & - & $3 \mathrm{~b} 2 \mathrm{c}$ & - & $R R E$ & $810 /-1$ \\
\hline & $143 \mathrm{~d}$ & $152 \mathrm{a}$ & $157 \mathrm{a}$ & $155 \mathrm{~b}$ & $159 \mathrm{~b}$ & $158 \mathrm{a}$ & $156 b$ & & 16 & & & $3 a 3 b$ & 35 & 27.7 & 0.00 \\
\hline & $125 \mathrm{~d}$ & & & & $121 \mathrm{~b}$ & $124 a$ & $122 b$ & $126 \mathrm{c}$ & 126.4 & 92 & - & $\mathrm{a} 2 \mathrm{~b}$ & $\mathrm{c}$ & $S V C$ & $8800 / 0$ \\
\hline & & & $136 \mathrm{c}$ & $135 \mathrm{c}$ & $139 \mathrm{~d}$ & & $121 b$ & & 18 & & & $\mathrm{~b} 2 \mathrm{c}$ & 17 & 15.8 & 0.00 \\
\hline & & $107 \mathrm{c}$ & $113 b$ & & & & & & 112.7 & 103 & - & $\mathrm{bc}$ & - & $S V C$ & $1215 /-4$ \\
\hline & $118 \mathrm{~d}$ & $116 \mathrm{~d}$ & $118 \mathrm{c}$ & & & & & & 11 & & & c & 5 & 11.5 & 0.20 \\
\hline \multirow[t]{3}{*}{$96 \mathrm{c}$} & & & & & $85 b$ & $90 a$ & & $84 a$ & 89.0 & 130 & $\mathrm{c}$ & $\overline{a b}$ & $\bar{a}$ & $\overline{S V C}$ & $4995 / 0$ \\
\hline & & & & $88 \mathrm{~d}$ & & $96 \mathrm{~b}$ & $83 b$ & & 13 & & & $2 \mathrm{~b}$ & 20 & 18.3 & 0.00 \\
\hline & $75 \mathrm{~b}$ & $76 b$ & $80 \mathrm{~b}$ & $80 \mathrm{~b}$ & & $74 \mathrm{~d}$ & $80 \mathrm{c}$ & & 77.4 & 150 & - & $4 \mathrm{bc}$ & - & $S V C$ & $1215 /-1$ \\
\hline $60 \mathrm{~d}$ & & $52 \mathrm{c}$ & $52 \mathrm{c}$ & $50 \mathrm{~b}$ & & $53 a$ & & & 13 & & & $\mathrm{ab} 2 \mathrm{c}$ & 15 & 23.5 & 0.00 \\
\hline
\end{tabular}

The first nine columns give frequencies of the lines found in the indicated type of data. The small letter following the frequency denotes the interval to which belongs the probability to obtain by chance as high a peak at this frequency as in the original periodogram. The intervals are as follows: "a": $<0.1 \%$ "b": $0.1-1 \%$, "c": $1-5 \%$, "d": $5-15 \%$. The bold letters indicate frequencies of the large peaks having $F A P$ values smaller than $0.5 \%$. Columns 10 and 11 give the mean frequency and mean period of the line. They are calculated only from the periods marked by letters "a", "b", "c". The number under the mean frequency is the difference between the highest and the lowest frequency from all the periods found. The next three Cols. 12-14 describe in a shorter way in what type of data (magnetic, radio, spot numbers) the given line is visible. We mark this using the letters "a", "b", "c", and two symbols "+" and "-". The symbol "-" means that the given line is not noticed in the respective type of data. The letters "a", "b", and "c" indicate that the line is recognised and the periods found have the probability level described by these letters, while the symbol "+" indicates that in the given type of data are periods having the probability level described by the letter "d". The second row in Col. 14 gives the importance number which indirectly measure how large is support for this line in all the analysed time series (see text). The last two columns in the first row, determine the time series in which the highest peak for the given line have been observed. The second row gives the normalized power of this peak as well as the resulting $F A P$ value.

complexes of activity "active longitudes" (Bumba \& Howard 1969; Ruzmaikin 1998) and its period may be identified with a fundamental period of unknow Sun's clock which value is freqeuntly taken as equal to 25.5 or 25. 8 (Bai \& Sturrock 1993; Bai 1994);

3. Activity lines: all lines with periods longer than 75 days. We propose this name as the strongest 151 days line in this group is thought to be related to the well known periodicity near 154 days seen mainly in the occurrence of high energy solar flares at the maximum of the solar sunspot activity. It is interesting to notice that this line is also observed in the minimum, but with a small importance number and only in the radio time series.

In the minimum, the distribution of the line periods in the investigated time window is much more uniform. There are 5 lines with periods shorter than 25 days but only one belongs to magnetic lines. Within an accuracy of \pm 0.3 all of them were noted before in various time series (Hughes \& Kesteven 1981; Pap et al. 1990). The lines with mean 
Table 5. Same as Table 4 but for the rising phase data.

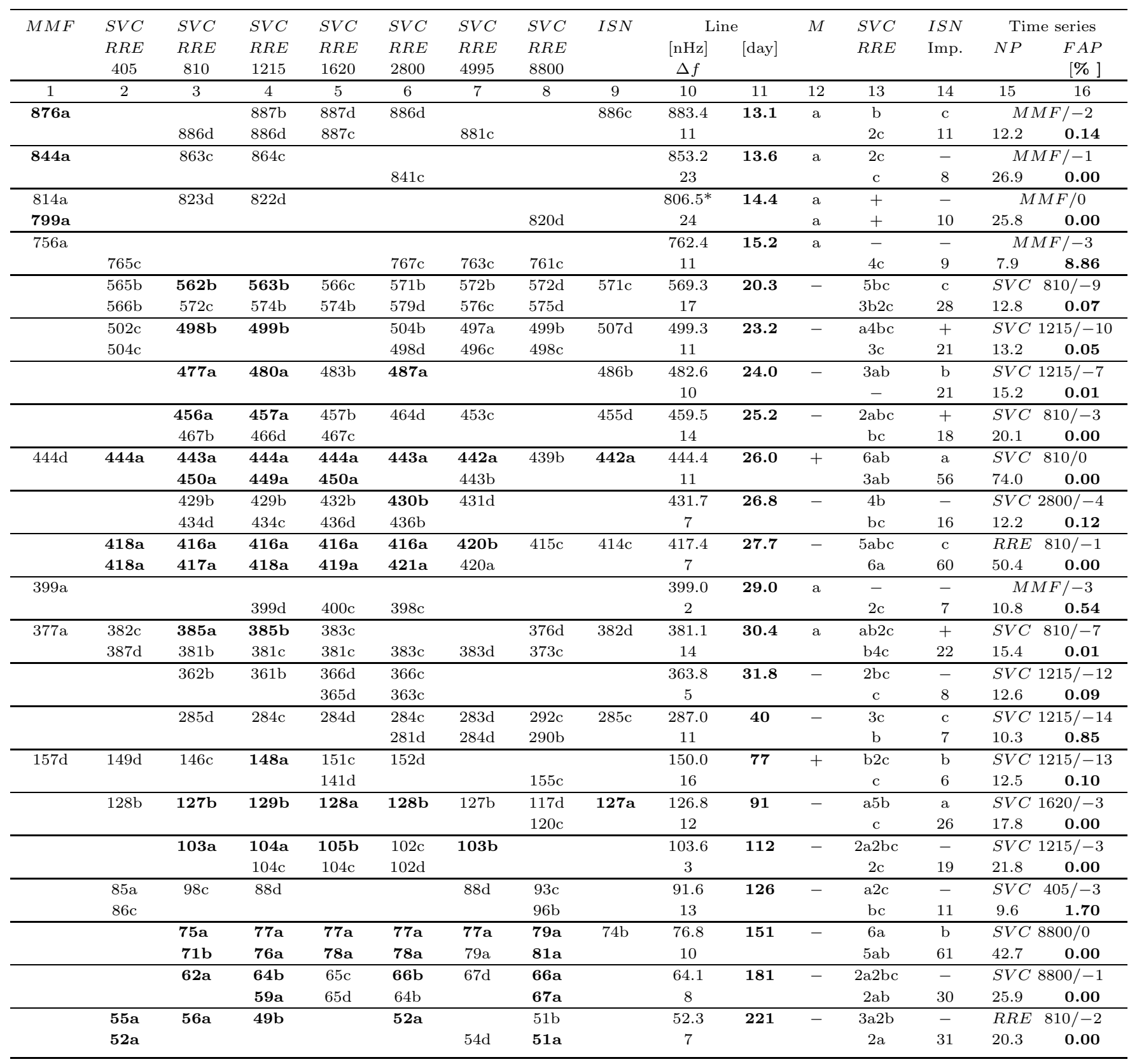

* This is the mean value from two close magnetic lines.

periods 13.6 and 23.2 are the most interesting. They belong to the group of 9 lines which are observed in both phases of the solar cycle investigated. Although in our data their importance numbers are not large, they were recognised as the dominant periods in the SMM/ACRIM total irradiance data for the years 1980-1988 (see Pap et al. 1990). A very intriguing group of lines is formed by 4 lines (39, 43, 48, 58 days) also observed in the minimum. All these lines are seen only in the time series which were obtained from the radio data, except for one with period 58 days also observed in the $I S N$ data. Among them only the line at 39 days is also present in the rising phase, although a small shift of its period is visible. Coming to longer periods we see that most of them are present in both investigated phase of the solar cycle. However, in the rising phase we observed more lines and all of them are stronger than those in the minimum. In the minimum, the strongest line in this group has period 75 days. However, in the rising phase this line is more weaker. It is not clear if it should be correlated with periods 73-78 days observed by some authors (Bai \& Sturrock 1991; Bai 1992a; Özgüç \& Ataç 1994) mainly in flare activity during the maximum of a solar cycle. We suppose that this line could be important if it is a real harmonic of the best known line with 
period near 154 days. It is important to notice that all of them were mentioned before in various analyses (Lean \& Brueckner 1989; Pap et al. 1990; Bai \& Sturrock 1991; Kile \& Cliver 1991; Antalová 1999).

Many previous studies by a number of authors have resulted in a wide range of solar periodicities, which are not easy to explain. This indicates that the problem of solar periodicities is still open and more systematic efforts should be undertaken. Here, we do not want to discuss all possible causes of the observed periods, but we want to present a suggestion which may be of help in further investigations.

Recently Oliver et al. (1998) proposed that the periodic emergence of magnetic flux, manifested as sunspots, triggers the near 158 day periodicity in high-energy solar flares. As different magnetic features have different rates of rotation (Gilman 1974; van Tend \& Zwaan 1976; Erofeev 1999) we think that a periodic emergence and a constant conversion of various magnetic structures explain the origin of the observed lines and their transformation with the phase of solar cycle 23. The main arguments supporting this idea are as follows:

1. There is a clear difference between the lines obtained in the minimum and in the rising phase. The lines characterictic for the minimum are probaly connected with both the short-lived small-scale magnetic fields which originate fairly high in the convective zone (Golub et al. 1981; Rivin 1999) and large-scale fields structures like coronal holes, coronal neutral sheet, global neutral lines. All these small and large scale magnetic fields dominate in the minimum phase of solar cycle (Golub et al. 1981; Wang et al. 1996; Lantos 1999);

2. In the rising phase a new distinct rotational line at 26.0 days is seen. This period is equal to the rotation rate of the active longitudes (zones) at $30^{\circ}$ (where the new flux appeared) determined from SOHO/MDI magnetograms by Benevolenskaya et al. (1999). They found in the northeren hemisphere the rotation rate $446.6 \pm 1.7 \mathrm{nHz}$ and $444.8 \pm 1.6 \mathrm{nHz}$ in the southern one, while our value for the whole Sun data is $444.4 \pm$ $4 \mathrm{nHz}$. Also the rotation rate of the equatorial zons equal $461.8 \mathrm{nHz}$ from SOHO observations is almost the same as the period $25.2(459.5 \mathrm{nHz})$ seen in our radio data (see Table 5);

3 . The line at 151 days is in the rising phase very prominent. This period is connected by Bogart (1982), Bai (1987) and Oliver et al. (1998) with strong, long-lived active regions giving most of the energetic solar events. This regions are generated by the emergence within the "active longitudes" magnetic tubes formed in the dynamo located at the base of the convection zone (Golub et al. 1981; Benevolenskaya et al. 1999; Rivin 1999). We also observe in the rising phase $I S N$ periodogram periods such as 13 . 1 or 23 . 8 , which were linked with the "active longitudes" and active centers in deep layers by Bai (1987).

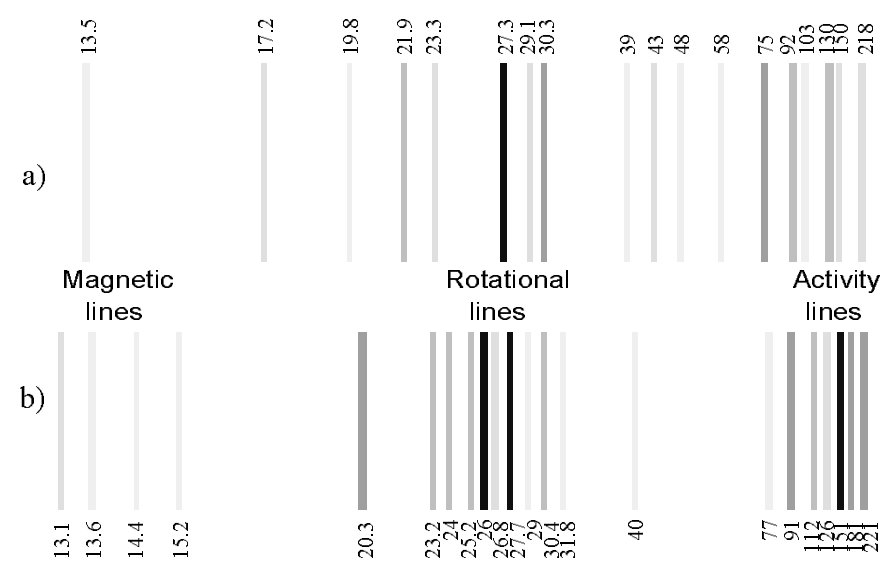

Fig. 8. Characteristic lines generated by solar phenomena in a) the minimum and $\mathbf{b}$ ) the rising phase of solar cycle 23 . The three group of lines are inicated: the level of darkness qualitatively illustrate the importance of lines.

\section{Conclusion}

To summarise, our analysis of radio observations gives the majority of the known periods, reveals a clear difference among periodicities observed in the two phase of the solar cycle examined and shows that daily measured radio fluxes at various frequencies are very useful for the systematic study of solar periodicities observed in the different layers of the solar atmosphere. We are preparing a similar analysis for the next phases of solar cycle 23: the maximum and the declining phase.

We found the rotation rate of the "active longitudes" in the rising phase as equal to $444.4 \pm 4 \mathrm{nHz}\left(26^{\mathrm{d}} .0 \pm\right.$ $0.3)$. We suppose that this period can be identified with a fundamental period of unknow Sun's clock as a lot of the known periodicities are subharmonics of it. We think that in the minimum the lines observed are conected with the small and large-scale magnetic fields which then dominate, while in the rising phase most lines are generated by new magnetic structures connected with long lived active regions formed within "active longitudes".

To understand the cause of all the observed periodicities, we still need new observational data, but more careful analyse of old radio data can also be useful (in preparation). However, our investigation, indicates that the solution of the observed solar periodicities should be sought in a complicated Sun's magnetic system which generate in the different solar data the compound set of solar periodicities.

Acknowledgements. First of all, we would like to thank our anonymous referee for very valuable remarks and suggestions which helped us to significantly improve the current version of the paper. The autors are grateful to Dr. K. Chyży for assistance in preparation of the manuscript. This work was supported in partly by KBN grant No. 158/E - 338/SPUB $204 / 93$. 


\section{References}

Antalová, A. 1999, in Proc. 9th European meeting on solar physics, Florence, Italy (ESA SP-448), Magnetic fields and solar processes, 743

Bai, T. 1987, ApJ, 314, 795

Bai, T. 1992a, ApJ, 388, L69

Bai, T. 1992b, ApJ, 397, 584

Bai, T. 1994, Solar Phys., 150, 385

Bai, T., \& Cliver, E. W. 1990, ApJ, 363, 299

Bai, T., \& Sturrock, P. A. 1991, Nature, 350, 141

Bai, T., \& Sturrock, P. A. 1993, ApJ, 409, 476

Ballester, J. L., Oliver, R., \& Baudin, F. 1999, ApJ, 522, L153

Benevolenskaya, E. E., Hoeksema, J. T., Kosovichev, A. G., \& Scherrer, P. H. 1999, ApJ, 517, L163

Bogart, R. S. 1982, Solar Phys., 76, 155

Bogart, R. S., \& Bai, T. 1985, ApJ, 299, L51

Bouwer, S. D. 1992, Solar Phys., 142, 365

Bumba, V., \& Howard, R. 1969, Solar Phys., 7, 28

Carbonell, M., \& Ballester, J. L. 1990, A\&A, 238, 377

Carbonell, M., \& Ballester, J. L. 1992, A\&A, 255, 350

Das, T. K., \& Chatterjee, T. N. 1996, MNRAS, 278, 6

Das, T. K., \& Nag, T. K. 1998, Solar Phys., 179, 431

Das, T. K., \& Nag, T. K. 1999, MNRAS, 303, 221

De Jager, O. C. 1987, Ph.D. Thesis, Potchefstroom University

Delache, P., \& Scherrer, P. H., 1983, Nature, 306, 651

Delache, P., Laclare, F., \& Sadsoud, H. 1985, Nature, 317, 416

Dennis, B. R. 1985, Solar Phys., 100, 465

Dröge, W., Gibbs, K., Grunsfeld, J. M., et al. 1990, ApJS, 73, 279

Erofeev, D. V. 1999, Solar Phys., 186, 431

Gilman, P. A. 1974, ARA\&A, 12, 47

Golub, L., Rosner, R., Vaiana, G. S., \& Weiss, N. O. 1981, ApJ, 243, 309
Horne, J. H., \& Baliunas, S. L. 1986, ApJ, 302, 757

Hughes, V. A., \& Kesteven, M. J. L. 1981, Solar Phys., 71, 259

Kile, J. N., \& Cliver, E. W. 1991, ApJ, 370, 442

Krüger, A. 1979, Introduction to solar radio astronomy and radio physics (Reidel, Dordrecht), 62

Lantos, P., Alissandrakis, C. E., \& Rigaud, D. 1992, Solar Phys., 137, 225

Lantos, P. 1999, in Proceedings of nobeyama symposium 1998 (NRO Report No. 479), Solar physics with radio observations, 11

Lean, J. L., \& Brueckner, G. E. 1989, ApJ, 337, 568

Lewis, D. J., Simnett, G. M., Brueckner, G. E., et al. 1999, Solar Phys., 184, 297

Oliver, R., \& Ballester, J. L. 1995, Solar Phys., 156, 145

Oliver, R., Ballester, J. L., \& Baudin, F. M. 1998, Nature, 394, 552

Özgüç, A., \& Ataç, T. 1994, Solar Phys., 150, 339

Pap, J., Tobiska, W. K., \& Bouwer, S. D. 1990, Solar Phys., 129,165

Rieger, E., Share, G. H., Forrest, D. J., et al. 1984, Nature, 312,623

Rivin, Yu. R. 1999, Solar Phys., 187, 207

Ruzmaikin, A. 1998, Solar Phys., 181, 1

Solar Geophysical Data, 1996-1999

Scargle, J. D. 1982, ApJ, 263, 835

van Tend, W., \& Zwaan, C. 1976, in IAU Symp. 71, Basic mechanisms of solar activity, ed. V. Bumba, \& J. Kleczek (Reidel, Dordrecht), 45

Wang, Y.-M., Havley, S. H., \& Sheeley, Jr. N. R. 1996, Sciences, 271,464

Zięba, S., Kułak, A., Machalski, J., Masłowski, J., \& Michalec, A. 1996, in Proc. VIII Nat. Symp. of radio science URSI '96, ed. W. Wolski (Oficyna Wydawnicza Politechniki Wrocławskiej, Wrocław), 233 